\title{
Laser Endoscopic Microsurgery of Laryngeal Cancers
}

\author{
Sereme Moustapha ${ }^{1}$ Dassonville Olivier ${ }^{1}$ Bozec Alexandre ${ }^{1} \quad$ Gilles Poissonnet $^{1}$ \\ 1 Department of ENT, Institut Universitaire de la Face et du Cou (IUFC), \\ Center Antoine Lacassagne, Nice, France \\ Int Arch Otorhinolaryngol 2014;18:36-38. \\ Address for correspondence Dr. Sérémé Moustapha, MD, Department \\ of ENT and Neck Clinical, Institut Universitaire de la Face et du Cou \\ (IUFC), Center Antoine Lacassagne. 31 Avenue Vallombrose: 06107 \\ Nice, France Cedex: 2 (e-mail: serememoustapha@yahoo.fr).
}

\begin{abstract}
Introduction The aim of our study is to present our experience in treating laryngeal cancers with endoscopic $\mathrm{CO}_{2}$ laser surgery.

Materials and Methods This retrospective study was conducted from January 2008 to December 2012 at Antoine Lacassagne Center of Nice. The functional and oncologic results of this surgical technique were analyzed and discussed.

Results The average length of hospital stay was 2.5 days. Phonatory results were considered excellent in $75 \%$ of our patients who maintained satisfactory phone communication skills after their interventions. Chronic hoarseness was the most common reason for bilateral or extended cordectomies. On the oncologic basis, histologic exam showed $90 \%$ of our margins were normal. The clinical control showed

Keywords

- laser therapy

- endoscopy

- laryngeal neoplasms excellent laryngeal preservation in $94.23 \%$. In 5 years, the overall survival was estimated at $96.15 \%$.

Conclusion This study shows the benefit of the laser endoscopic microsurgery for the preservation of laryngeal functions and the local histologic control, essentially for early stage laryngeal cancers.
\end{abstract}

\section{Introduction}

Conservative surgery of vocal cord pathology combines the use of an endoscopic route, a surgical microscope, and a $\mathrm{CO}_{2}$ laser. Strong and Jako ${ }^{1}$ described this surgical technique for the first time in $1970,{ }^{2}$ which experienced a strong growth in popularity over the past 20 years under the leadership of several authors. ${ }^{3,4}$

The aim of our study was to evaluate the functional and oncologic results of our patients operated by this technique of laser endoscopic microsurgery.

\section{Materials and Methods}

From January 2008 to December 2012, 52 patients followed for laryngeal cancer were treated with $\mathrm{CO}_{2}$ laser endoscopic microsurgery in our center. There were 47 men and 5 women of average age 63.5 years (range 52 to 81 ).
Chronic hoarseness (39 cases) was the essential reason for consultation with an average duration of 4 months. The examination found a history of smoking in 38 cases, alcoholic use in 21 cases, and previous irradiation in 12 cases. All of our patients were examined thoroughly (physical examination + direct flexible laryngoscopy) and systematically (with a panendoscopy and computed tomography scan).

The nature of the tumor was determined by the extemporaneous histologic reading of the piece after endoscopic biopsy. Later confirmation was given by histologic examination. There were 52 cases of squamous cell carcinoma.

All our interventions were performed under general anesthesia with oral intubation. The surgical instruments consisted of endoscopic and microscopic surgery equipment, a surgical microscope, and laser tubes of $\mathrm{CO}_{2}$. We used a cordectomy for cancer with a glottic location, and for supraglottic cancer, a supraglottic laryngectomy dissection-type epiglottectomy with a systematic dissection of neck (type III).

Copyright $(2014$ by Thieme Publicações License terms

Ltda, Rio de Janeiro, Brazil

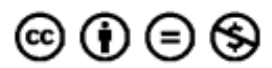

August 9, 2013

accepted

September 17, 2013
DOI http://dx.doi.org/

10.1055/s-0033-1361084. ISSN 1809-9777. 
The attending physician followed all our patients regularly for 1 to 2 weeks after surgery. Later, the clinical condition of the patient determined the frequency of the follow-up, varying between 2 and 4 months for the first and the second year. This follow-up consisted of a thorough clinical examination coupled with systematic indirect endoscopies and computed tomography scans.

Our patients were systematically sent to a physiotherapist for vocal and sometimes nutritional rehabilitation. The functional outcome was evaluated based on the duration of hospitalization, need for tracheotomy/ nasogastric tube, and quality of swallowing and speech. In accordance with Li-Jen et al, ${ }^{5}$ the evaluation of speech depended on the patient's ability to maintain a proper telephone communication within 1 month after the intervention.

Our oncologic evaluation criteria took into account the resection margins and cuttings, local control, laryngeal preservation, completion of a complementary treatment (radiation and/or chemotherapy), local and lymph node recurrence, and mortality.

\section{Results}

Systematically, our patients benefited from a thorough clinical examination (physical examination + direct flexible laryngoscopy), panendoscopy, and computed tomography. These explorations have highlighted 39 glottic locations, 10 supraglottic locations, 3 glottic and supraglottic locations, 2 cases of cervical ganglion flooding, and 1 esophageal secondary location.

According to the Union for International Cancer Control/ American Joint Cancer Committee's 2002 laryngeal cancer classification, our carcinomas were classified as Tis in 9 cases, $\mathrm{T} 1$ in 35 cases, T1b in 5 cases, and T2 in 3 cases.

For glottic locations according to the European Society of Laryngology classification, ${ }^{2,5}$ our cordectomies were type I (epithelial) in 7 cases, type III (transmuscular) in 29 cases, type $\mathrm{Va}$ (extended to the anterior commissure) in 3 cases, and type Vc (extended to the supraglottic area) in 3 cases. Our 10 supraglottic locations benefited from a supraglottic laryngectomy dissection-type epiglottectomy with a systematic neck modified radical dissection (type III).

\section{Functional Results}

The average length of hospitalization of our patients was 2.5 days (range 1 day in the cordectomy type I and III, 7 to 18 days in the extended cordectomy type Va and Vc). Disorders of swallowing corresponding to the type of false roads were noted in 24 patients. In 7 of these patients, placement of a nasogastric tube for nutrition was necessary. A tracheotomy was performed in emergency on 1 patient in the emergency room for severe postsurgical laryngeal shortness of breath. The phonatory results have been satisfactory, with 34 patients $(75 \%)$ maintaining phone communication skills in the months following the intervention. The trailing hoarseness was especially encountered in our patients who benefited from a bilateral or extensive cordectomy (type: Va, Vc). Voice quality was considered good in all our patients by the physiotherapist at the end of the voice rehabilitation.

\section{Oncologic Results}

In $90 \%$ (47 cases) of our resections, margins were healthy. In one case, the endoscopic resection corrected the histologic biopsy diagnosis: one mucoepidermoid carcinoma was in reality a squamous cell carcinoma.

The 5-year survival was estimated in our series to be 96.15\%. We had two deaths: one patient died 2 months postoperatively of an array of cardiovascular decompensation and esophageal metastasis and high blood pressure. The other died of cancer at 14 months after the procedure.

The local oncologic control was $94.23 \%$; our three relapses have all occurred on previously irradiated areas and the patients have gone on to total laryngectomy.

\section{Discussion}

Functional surgery of laryngeal cancers has long been done externally in the form of partial surgery and by radiotherapy more recently. The introduction of laser endoscopic microsurgery has revolutionized this treatment, as well as had tremendous effect on the duration of hospitalization, swallowing and speech disorders, and need for tracheotomy and/ or nasogastric tube.

All the authors agree on a significant reduction in the length of hospital stay after laser endoscopic surgery. According to Brasnu $^{2}$ and Ansarin et al, ${ }^{6}$ the length of hospital stay was reduced from 1 to 3 days. The average length of hospitalization in our study was 2.5 days. Longer stays of hospitalization in our series were for extended cordectomies ( Va, Vc) or supraglottic laryngectomies due to more frequent postoperative complications. The practice of a tracheotomy is exceptional after laser endoscopic surgery. ${ }^{2,7,8}$ Moreau and Pierre used laser to treat invasive or in situ laryngeal carcinoma in 160 patients, doing no tracheotomy postoperatively. ${ }^{7}$ In our series, a single postoperative tracheotomy was necessary, largely due to infectious complication (compressive cervical abscesses). In the series of Policarpo et $\mathrm{al}^{9}$ and Moreau and Pierre, ${ }^{7}$ as in ours, postsurgery voice quality depended on the type of cordectomy. The voice quality was worse if cordectomy was extended (bilateral type III, type Va, and type Vc). By comparing the quality and voice recovery time after laser endoscopy and after radiotherapy, Christine et al concluded that voice quality was normal 3 months after laser cordectomy. ${ }^{10}$ However, after radiotherapy, this standardization takes longer and the two qualities of voice will be equivalent in the long run.

Swallowing is generally preserved after laser endoscopic microsurgery. Brasnu ${ }^{2}$ explained rapid swallowing recovery by the recovery of an efficient glottic closure reflex 48 to 72 hours after the laser surgery, although after an externally supraglottic laryngectomy, the glottic closure reflex is inefficient for at least 3 weeks. In our series, 24 patients (46.15\%) presented food false routes but only 7 of them (13.46\%) required the establishment of a nasogastric tube. Ruben et al compared the length of time of nasogastric feeding after a laser supraglottic laryngectomy to that of tube feeding after an external supraglottic laryngectomy and found an average of 9.53 days for laser laryngectomy and 16.95 days for external surgery. ${ }^{11}$ 
Local control and laryngeal preservation after laser endoscopic microsurgery are regarded as excellent by many authors. $^{2,5,6,9}$ Preuss et al, with a 5-year follow-up period, had a local control rate ranging from 94.6 to $98.2 \%$ and a rate of laryngeal preservation between 95.5 and $100 \%$. In our series, the local control rate was $94.23 \%$ in 5 years. ${ }^{12}$

The assessment of local control based on the invasion or not of anterior commissure is not yet the object of consensus. According to Peretti et a ${ }^{13}$ and Pearson and Salassa, ${ }^{14}$ statistically there is no significant difference of local control in accordance with the flooding or not of the anterior commissure. However, Steiner et al found that local control was not as good in the event of infringement of the anterior commissure. ${ }^{3}$

Our study did not find any statistical difference but noted a greater difficulty of exposure when the tumor extended to the anterior commissure. Also in our practice, we limited the indications of laser endoscopic surgery to cancers of the larynx classified as Tis, $\mathrm{T} 1$, and T2. In the literature, there is not a generally agreed upon opinion on the indications of laser endoscopic surgery of larynx cancers. ${ }^{3,8,9,12}$

The assessment of local control of laryngeal preservation and of the survival according to the type of treatment shows that there is no significant difference between external conservative surgery or laser surgery and radiotherapy. 2,5,9 However, for Brasnu, ${ }^{2}$ the advantage of the laser endoscopic surgery is to avoid incision of skin and platysma, to preserve normal tissue, to keep swallowing and speech functions, and to avoid the side effects of radiotherapy.

Laser endoscopic surgery is still nowadays one of the most efficient techniques of preservation of laryngeal functions despite the introduction in recent years of the robot-assisted transoral surgery, which is not widely used.

\section{Conclusion}

The concern of any practitioner is the constant improvement in patient care. Laser laryngeal endoscopic surgery lies within this framework with significant reduction of postoperative complications. It also allows for local control, laryngeal preservation, better voice quality, and survival equivalent to that of the external surgery and radiotherapy, hence the interest of this surgical technique in the early support of laryngeal cancers.

\section{References}

1 Strong MS, Jako GJ. Laser surgery in the larynx. Early clinical experience with continuous CO2 laser. Ann Otol Rhinol Laryngol 1972;81:791-798

2 Brasnu D. Chirurgie conservatrice des cancers du larynx: de la chirurgie partielle par voie cervicale à la chirurgie minimale invasive. E-mémoires de l'Académie Nat de Chir 2010;9(4):77-81

3 Steiner W. Results of curative laser microsurgery of laryngeal carcinomas. Am J Otolaryngol 1993;14(2):116-121

4 Remacle M, Lawson G, Jamart J, Minet M, Watelet JB, Delos M. CO2 laser in the diagnosis and treatment of early cancer of the vocal fold. Eur Arch Otorhinolaryngol 1997;254(4):169-176

5 Li-Jen H, Tuan-Jen F, Kai-Ping Ch, et al. Transoral endoscopic CO2 laser microsurgery for early laryngeal cancers. Chang Gung Med J 2009;32(5):517-525

6 Ansarin M, Cattaneo A, Santoro L, et al. Laser surgery of early glottic cancer in elderly. Acta Otorhinolaryngol Ital 2010;30(4):169

7 Moreau PR, Pierre R. Treatment of laryngeal carcinomas by laser endoscopic microsurgery. Laryngoscope 2000;110(6):1000-1006

8 Bumber Z, Prgomet D, Janjanin S. Endoscopic CO2 laser surgery for supraglottic cancer-ten years of experience. Coll Antropol 2009; 33(1):87-91

9 Policarpo M, Aluffi P, Brovelli F, Borello G, Pia F. Oncological and functional results of $\mathrm{CO} 2$ laser cordectomy. Acta Otorhinolaryngol Ital 2004;24(5):267-274

10 Christine van Gogh DL, Irma verdonck-de leeuw M, Jeanne WedlerPeeters et al. Prospective evaluation of voice outcome during the first two years in male patients treated by radiotherapy or laser surgery for T1a glottic carcinoma. Eur Arch Otorhinolaryngol 2012;269(6):1647-1652

11 Ruben C, Juan PR, Jose LL, Vanessa S, Primitivo O, Carlos S. Functional Outcomes of Transoral Laser Surgery of Supraglottic Carcinoma Compared with a Transcervical Approach. Asturias: Wiley Interscience; 2004:653-659

12 Preuss SF, Cramer K, Klussmann JP, Eckel HE, Guntinas-Lichius O. Transoral laser surgery for laryngeal cancer: outcome, complications and prognostic factors in 275 patients. Eur J Surg Oncol 2009; 35(3):235-240

13 Peretti G, Nicolai P, Redaelli De Zinis LO, et al. Endoscopic CO2 laser excision for tis, T1, and T2 glottic carcinomas: cure rate and prognostic factors. Otolaryngol Head Neck Surg 2000;123(1 Pt 1):124-131

14 Pearson BW, Salassa JR. Transoral laser microresection for cancer of the larynx involving the anterior commissure. Laryngoscope 2003;113(7):1104-1112 